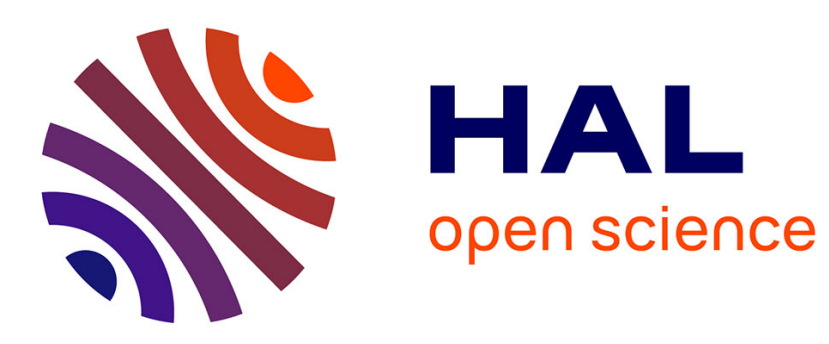

\title{
Flexible Virtual Structure Consideration in Dynamic Modeling of Mobile Robots Formation
}

\author{
Asma Essghaier, Lotfi Beji, Jean Lerbet, Azgal Abichou
}

\section{To cite this version:}

Asma Essghaier, Lotfi Beji, Jean Lerbet, Azgal Abichou. Flexible Virtual Structure Consideration in Dynamic Modeling of Mobile Robots Formation. 2nd Mediterranean Conference on Intelligent Systems and Automation (CISA 2009), Mar 2009, Zarzis, Tunisia. pp.296-301, 10.1063/1.3106489 . hal-00668050

\section{HAL Id: hal-00668050 https://hal.science/hal-00668050}

Submitted on 6 Jan 2020

HAL is a multi-disciplinary open access archive for the deposit and dissemination of scientific research documents, whether they are published or not. The documents may come from teaching and research institutions in France or abroad, or from public or private research centers.
L'archive ouverte pluridisciplinaire HAL, est destinée au dépôt et à la diffusion de documents scientifiques de niveau recherche, publiés ou non, émanant des établissements d'enseignement et de recherche français ou étrangers, des laboratoires publics ou privés. 


\title{
Flexible Virtual Structure Consideration in Dynamic Modeling of Mobile Robots Formation
}

\author{
A. Essghaier El Kamel ${ }^{* * *}$ L. Beji. ${ }^{* *}$ J. Lerbet ${ }^{* *}$ A. Abichou ${ }^{*}$ \\ * Mathematical Engineering Laboratory, Tunisia Polytechnic School, \\ (e-mail: Asma.Essghaier, Azgal.Abichou@ept.rnu.tm) \\ ** Informatics, Integrative Biology and Complex Systems, 40 Rue de Pelvoux; \\ 91020 Evry Cedex; France \\ (e-mail: Asma.elkamel,Lotfi.Beji,Jean.Lerbet@iup.univ-evry.fr).
}

\begin{abstract}
In cooperative mobile robotics, we look for formation keeping and maintenance of a geometric configuration during movement. As a solution to these problems, the concept of a virtual structure is considered. Based on this idea, we have developed an efficient flexible virtual structure, describing the dynamic model of $n$ vehicles in formation and where the whole formation is kept dependant. Notes that, for $2 D$ and $3 D$ space navigation, only a rigid virtual structure was proposed in the literature. Further, the problem was limited to a kinematic behavior of the structure. Hence, the flexible virtual structure in dynamic modeling of mobile robots formation presented in this paper, gives more capabilities to the formation to avoid obstacles in hostile environment while keeping formation and avoiding inter-agent collision.
\end{abstract}

Keywords: Formation keeping, Multi-agent, Flexible, Virtual structure.

\section{INTRODUCTION}

$\mathbf{I}_{\mathrm{i}}^{\mathrm{N}}$

Nterest to mobile robots formation and their control have increased because of the large applications in military as well as civil field. The study of robot formation control, inspired from swarms evolution in nature, began from the industry and military worlds with the idea of using multiple small vehicles instead of one big. Teams of inexpensive robots, performing cooperative tasks, may prove to be more cost and energy effective than a single one. They are capable, as well, to achieve a mission more efficiently. Using formations of robots includes more advantages as increased feasibility, accuracy, robustness, flexibility, and probability of success.

Recently, many works have focused on the subject, based on different approaches and using different strategies. Three main ones can be distinguished: virtual structure, behavioral and leader following approach. Each approach have its advantages and disadvantages, and is used in order to achieve a specific goal. Adopting one method or another depends as well on the choice of a centralized or decentralized approach. The leader following approach is a centralized approach since all the agents of the formation depend on a leader which is the only decision maker of the group. This can be useful in some situations, where decentralized operations can represent a potential peril especially when adopted in the wrong context or situation, as it can be a critical choice, for example if the leader breaks down. Behavioral or behavior based approach deals with the behavior of the solution, it is a very efficient method but is generally more complicated than the other approaches. As an example of this approach we can cite works in [2].

Given a number of mobile robots, a solution to the problem of moving in formation must simultaneously satisfy two goals: making progress in a given direction and maintaining geometric compliance to the virtual structure imposed at all time.
The virtual structure approach, has been largely used in bibliography as can be presented in the previous work.

In [1], Lewis et al. developed a general control strategy to force an ensemble of robots to behave as if they were particles embedded in a rigid structure.

In [4], Monteiro et al. propose a dynamical systems approach to behavior-based formation control of three mobile robots. Behavioral variables are used to describe, quantify and internally represent the state of each robot (a master and two slaves), with respect to elementary behaviors, for target acquisition and obstacle avoidance. Only kinematics behavior are taken into account.

A formation control ideas for multiple spacecraft using virtual structure approach are presented by Ren in [3], authors maintain the stability of each spacecraft in formation in considering a feedback from spacecraft to the virtual structure. An extension to a decentralized scheme for spacecraft formation flying via the virtual structure approach is also proposed by Ren in [6]. The formation of vessels is treated in [5], where individual parameterized paths for each vessel is build so that when the parametrization variables are synchronized, the vessels are in formation. The control objective for each vessel is to maintain its position in the formation configured around a formation reference point which tracks a predefined path.

The work of Lalish [8] addresses an approach to 3D formation tracking control based on virtual structures. The virtual structure specifies the formation position of each vehicle, and virtual leaders are constructed ahead of each position for the vehicles to follow. In order to prevent transients from leading to collisions, deconfliction techniques are applied.

In [6], for spacecraft formation flying, a virtual structure that moves as a rigid body is assigned to the formation and each vehicle is given a set of coordinates to follow.

In this work, from the modeling point of view in mechanics, 
one brings a rigorous work to the system equations. Further. we build a new virtual structure which is flexible and able to describe the behavior of all the formation. This virtual structure, with a flexible character, ensures the formation expansion and contraction along the two cartesian axes. The result will also guarantee formation keeping over time. We have developed a linear dynamic equations of motion which will ease the formation control. This can be seen for example in some scenarios similar to the situation described in see Fig. 2.

The paper is organized as follows. Section 2 describes the problem and explains our motivation. Section 3 develops equations of motion of a system of $n$ mobile robots (agents) constrained to move in only one dimension. The equations of motion are developed and a strategy of formation's size extending is presented in section 4 . Section 5 shows that our approach is applicable for any geometric configuration both analytically and in simulation.

\section{PROBLEM DESCRIPTION}

Robot formation control include the control of relative distance between each two agents of the formation and how to combine at the same time no collision and formation keeping. This distance must be able to expand and regress (Fig. 1). In our approach, we model this behavior by a spring-damper element which will reproduce the contraction and expansion behavior while the addition of the damper will reduce any generated oscillation.

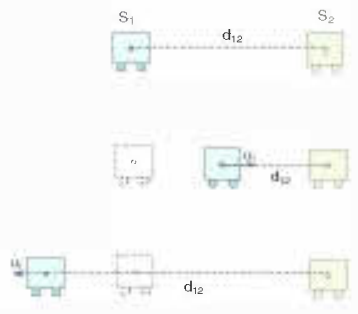

Fig. 1. Two agents relative distance

Vehicles are modeled as unconstrained mass particles under the influence of control forces in an inertial reference frame.

Virtual rigid structure approach is based on the idea of assigning a virtual rigid configuration to the formation. From the movement of the virtual structure, considered as a rigid body, we get a set of point that each vehicle has to track. This approach guarantees that no collision can occur between the different agents of the formation during movement. However, this approach is not very useful in hostile environments in presence of obstacles. Our main issue is to develop a flexible virtual structure that in addition to non inter-agent collision. guarantees to each vehicle the avoidance of obstacles in an unknown environment (see Fig.2).

\section{ONE DIMENSIONAL FORMATION}

In this section, we apply our approach to a formation of agents constrained to move in one dimension and will develop the relative equations of motion. Caicedo treated in [7] the one dimensional case and developed equations of motion for a

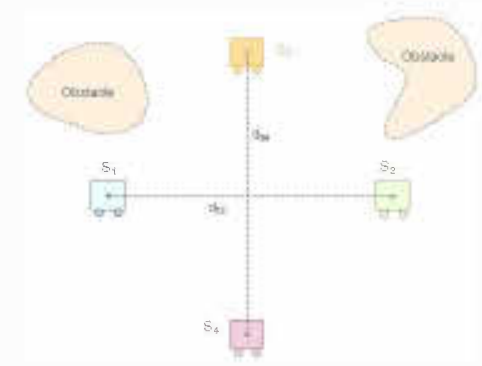

(a) A formation of 4 agents in front of obstacles

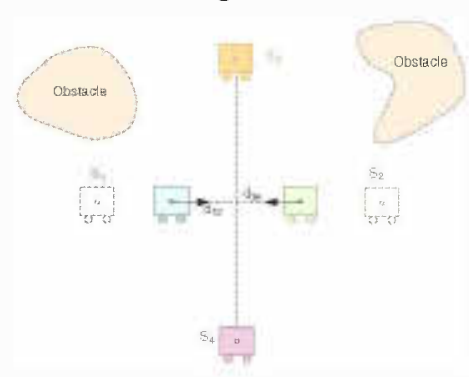

(b) Formation Contraction to avoid obstacles

Fig. 2. Contraction of formation while avoiding obstacle

rigid configuration. Here, we are interested to a flexible one dimensional formation.

Consider a system $\Sigma=\left\{S_{1}, \ldots, S_{n}\right\}$ of $n$ agents (rigid bodies), respectively, of masses $m_{1}, \ldots, m_{n}$ constrained to move in only one direction along $\vec{i}$. Note $G_{i}$ the center of gravity of each agent $S_{i}$. Each body $S_{i}$ is subject to an external force $u_{i}$ in the same direction $\vec{i}$. The $S_{i}$ are linked together by $(n-1)$ virtual flexible links, spring-damper like element, as following.

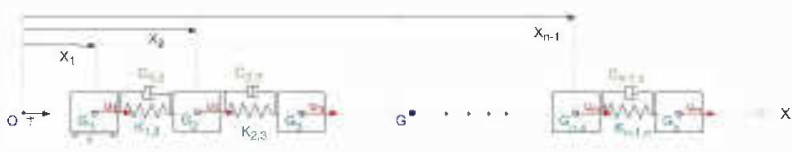

Fig. 3. Formation of $n$ agents constrained to move in one dimension

In reality there is not any link between the agents but these virtual links are taken into account in the formation's mechanical modeling. Indeed they obey to some assumptions:

- virtual links are massless.

- each virtual link is assimilated to a point.

Definition 1. The system $\Sigma$ is in equilibrium when all the spring damper elements are at their equilibrium length and the formation is in the desired configuration. Vehicles are then at their equilibria positions $\vec{X}_{i}=\overrightarrow{O G}_{i}=X_{i, e} \vec{i}$.

Let $X_{i}$, the position of robot $i$. We choose to identify $S_{i}$ by their displacement from their equilibria positions : $x_{i}=X_{i}-X_{i, e}$.

To write the formation's equations of motion, we define first a Lagrange's coordinate system :

$$
(\boldsymbol{t}, \mathbf{q})=\left(t, q_{1}, q_{2}, \ldots, q_{n}\right)=\left(t, X_{G}, x_{1,2}, \ldots, x_{1, n}\right)
$$


where $X_{G}$ is the formation's center of mass, defined by

$$
X_{G}=|\bar{O} \vec{C}|=\frac{1}{m} \sum_{i=1}^{n} m_{i}\left(x_{i}+X_{i, e}\right), m=\sum_{i=1}^{n} m_{i}
$$

And, for $i=2, \ldots, n$

$$
q_{i}=x_{i, i-1}=x_{i}-x_{i-1}
$$

In other way :

$$
\mathbf{q}=\mathbf{T x}+\mathrm{c}
$$

with :

$\mathrm{q}=\left(q_{1}, q_{2}, \cdots, q_{n}\right)^{t}, \mathrm{x}=\left(x_{1}, x_{2}, \cdots, x_{n}\right)^{t}$,

$\mathrm{c}=\left(\begin{array}{l}\frac{1}{m} \sum_{i=1} m_{\imath} X_{i, e} \\ 0 \\ \vdots \\ 0\end{array}\right)$ and $\mathbf{T}=\left(\begin{array}{ccccc}\frac{m_{1}}{m} & \frac{m_{2}}{m} & \frac{m_{3}}{m} & \ldots & \frac{m_{n}}{m} \\ -1 & 1 & 0 & \ldots & 0 \\ 0 & -1 & 1 & \ldots & \vdots \\ \vdots & \vdots & \ddots & \ddots & 0 \\ 0 & 0 & \ldots & -1 & 1\end{array}\right)$

The virtual work, largely known in mechanics, can be considered as a time integral of virtual power. The principle of virtual power is considered to derive the dynamic model of the formation. This leads to the following three steps.

1- Calculation of the virtual power of inertia :

$$
\mathscr{P}_{i, i n}^{*}=\dot{\mathrm{q}}^{* t}\left(-\frac{d}{d t}\left(\frac{\partial C}{\partial \dot{\mathbf{q}}}\right)+\frac{\partial C}{\partial \mathrm{q}}\right)
$$

The kinetic energy of the system is :

$$
C=\frac{1}{2} \sum_{i=1}^{n} m_{i} C_{i}=\frac{1}{2} \sum_{i=1}^{n} m_{i}\left\|\vec{V}\left(G_{i} / R_{0}\right)\right\|^{2} .
$$

Knowing that $\vec{V}\left(G_{i} / R_{0}\right)=\dot{X}_{i} \vec{i}=\dot{x}_{i} \vec{i}$ means that:

$$
C=\frac{1}{2} \sum_{i=1}^{n} m_{i} \dot{x}_{i}^{2}=\frac{1}{2} \dot{\mathbf{x}}^{t} \mathbf{M} \dot{\mathrm{x}}
$$

where $\mathbf{M}=\operatorname{diag}\left(m_{1}, m_{2}, \ldots, m_{n}\right)$.

Using the derivative of equation (4)with respect to time leads to the expression of $C$ in the coordinate system $q$ :

$$
C=\frac{1}{2}\left(\mathbf{T}^{-1} \dot{\mathbf{q}}\right)^{t} \mathbf{M T}^{-1} \dot{\mathbf{q}}=\frac{1}{2} \dot{\mathbf{q}}^{t} \widehat{\mathbf{M}} \dot{\mathbf{q}} \text {. }
$$

where $\widetilde{\mathbf{M}}=\mathrm{T}^{-\mathbf{1}^{t}} \mathbf{M T}^{-1}$.

Hence,

$$
\left\{\begin{array}{l}
\frac{\partial C}{\partial \boldsymbol{q}}=0 \\
-\frac{d}{d t}\left(\frac{\partial C}{\partial \dot{q}}\right)=-\frac{d}{d t}(\widehat{\mathbf{M}} \dot{q})=-\widehat{\mathbf{M}} \ddot{\mathbf{q}}
\end{array}\right.
$$

Finally we get

$$
\mathscr{P}_{i, i n}^{*}=-\dot{\mathrm{q}}^{* t} \widetilde{\mathbf{M}} \ddot{\mathrm{q}}
$$

2- Calculation of the virtual power of external actions, leads to

$$
\mathscr{P}_{e x t}^{*}=\sum_{i=1}^{n} \mathscr{P}_{i, e x t}^{*}=\sum_{i=1}^{m}\left[\mathcal{T}_{i, e x t}^{-} / V_{i}^{*}\right]
$$

Assume that each vehicle $S_{i}$ is subject to an external action $\vec{u}_{i}$ along the $x$ axis i.e $\vec{u}_{i}=u_{i} \vec{i}$.
Hence

$$
\mathscr{P}_{\text {ext }}^{*}=\sum_{i=1}^{n}\left[\mathcal{T}_{i, \text { ext }}^{-} / V_{i}^{*}\right]=\sum_{i=1}^{n} u_{i} \dot{x}_{i}^{*}
$$

Let $u_{X}=\left(u_{1}, \ldots, u_{n}\right)^{t}$. Hence, in other words, we have:

$$
\mathscr{P}_{\text {ext }}^{*}=\mathbf{u}_{\mathbf{X}} \mathbf{x}^{*}=\dot{\mathbf{q}}^{* t} \mathbf{T}^{-1^{t}} \mathbf{u}_{\mathbf{X}}
$$

We now turn to the last step necessary to write the equations of motion:

3- Calculation of the internal forces virtual power:

As previously mentioned, in our case virtual links are considered, however, they are involved in writing dynamic equations of the system.

Assume the existence of $n-1$ virtual links linking $S_{1}$ to $S_{2}$, $S_{2}$ to $S_{3}, \ldots$ and $S_{n-1}$ to $S_{n}$.

Let $V_{i}^{*}$ A virtual velocity field kinematically feasible at constant time for $i=1, \ldots, n$.

The expression of the internal forces virtual power is given by

$$
\mathscr{P}_{l}^{*}=\sum_{i=1}^{n} \sum_{j \neq i}^{n}\left[\mathcal{T}_{i, j} / V_{i}^{*}\right]
$$

As we need only links between two successive agents $\left(l_{i, j}=\left\{l_{i, i-1}, l_{i, i+1}\right\}\right)$ therefore the expression of the internal forces virtual power will be limited to:

$$
\mathscr{P}_{l}^{*}=\sum_{i=2}^{n}\left[\mathcal{T}_{i, i-1} / V_{i}^{*}-V_{i-1}^{*}\right]
$$

Before attempting the explicit expression of the internal forces virtual power, we will do a balance sheet of internal forces of our system:

$\forall i=2, \ldots, m$ we have two actions:

- recall action of the spring of link $l_{i, i-1}$.

- reaction of the viscous damper of link $l_{i, i-1}$.

Thus we have

$$
\mathscr{P}_{l}^{*}=\sum_{i=2}^{n} \mathscr{P}_{l_{i, i-1, s p r i n g}^{*}}^{*}+\mathscr{P}_{l_{i, i-1, \text { damper }}^{*}}
$$

Moreover, as $l_{i, i-1, s p r i n g}$ derive from a potential, we get

$$
\begin{aligned}
\mathscr{P}_{l_{i, i-1, s p r i n g}}^{*} & =\left[\mathcal{T}_{i, i-1, \text { spring }}^{-} / V_{i}^{*}-V_{i-1}^{*}\right] \\
& =-\sum_{k=1}^{n} \frac{\partial U_{i, i-1}(t, \mathbf{q})}{\partial q_{k}} \dot{q}_{k}{ }^{*}
\end{aligned}
$$

where $U_{i, i-1}$ is given by

$$
U_{i, i-1}=\frac{1}{2} K_{i, i-1} q_{i}^{2} .
$$

Hence,

$$
\mathscr{P}_{l_{i, i-1, s p r i n g}^{*}}^{*}=-K_{i, i-1} q_{i} \dot{q}_{i}^{*}
$$

On the other hand

$$
\mathscr{P}_{l_{i, i-1, d a m p e r}^{*}}=\left[\mathcal{T}_{i, i-1, \text { damper }} / V_{i}^{*}-V_{i-1}^{*}\right]
$$

The expression of $\mathcal{T}_{i, i-1, \text { damper }}$ is given by

$$
\mathcal{T}_{i, i-1, \text { damper }}=\left\{\begin{aligned}
\overrightarrow{\mathcal{R}} & =-C_{i, i-1}\left(\dot{X}_{i}-\dot{X}_{i-1}\right) \vec{i} \\
& =-C_{i, i-1} \dot{q}_{i} \vec{i} \\
\overrightarrow{\mathcal{M}} & =\overrightarrow{0}
\end{aligned}\right.
$$

And virtual velocity fields kinematically feasible at constant time associated, respectively with $S_{i}$ and $S_{i-1}$ conduct to:

$$
V_{i}^{*}-V_{i-1}^{*}=\left\{\begin{array}{l}
\overrightarrow{0} \\
\left(\dot{x}_{i}^{*}-\dot{x}_{i-1}^{*}\right) \vec{i}=\dot{q}_{i}{ }^{*} \vec{i}
\end{array}\right.
$$


This leads us to:

Finally, we get

$$
\mathscr{P}_{l_{i, i-1, \text { damper }}^{*}}=-C_{i, i-1} \dot{q}_{i} \dot{q}_{i}{ }^{*}
$$

$$
\mathscr{P}_{l}^{*}=\sum_{i=2}^{n}-\left(K_{i, i-1} q_{i}+C_{i, i-1} \dot{q}_{i}\right) \dot{q}_{i}^{*}
$$

This brings to the following equivalent equation :

$$
\mathscr{P}_{l}^{*}=\dot{\mathbf{q}}^{* t}(-\mathbf{K} \mathbf{q}-\mathbf{C} \dot{\mathbf{q}})
$$

According to the principle of virtual powers, we have, for every virtual velocity field kinematically feasible at constant time,

$$
\mathscr{P}_{i n}^{*}+\mathscr{P}_{e x t}^{*}+\mathscr{P}_{l}^{*}=\mathbf{0}, \forall \dot{\mathbf{q}}^{*}
$$

Finally,

$$
\widehat{\mathbf{M}} \ddot{\mathrm{q}}+\mathbf{K} \mathbf{q}+\mathbf{C} \dot{q}=\mathbf{T}^{-1^{t}} \mathbf{u}_{X}
$$

with :

$$
\mathbf{K}=\left(\begin{array}{cccc}
\bullet & \bullet & \cdots & \bullet \\
\bullet & \bullet & \ddots & \bullet \\
\bullet & \bullet & \ddots & K_{n, n-1}
\end{array}\right) \text { and } \mathbf{C}=\left(\begin{array}{cccc}
\bullet & \bullet & \cdots & 0 \\
0 & C_{2,1} & \cdots & 0 \\
\bullet & \bullet & \ddots & 0 \\
\bullet & \bullet & \ddots & C_{n, n-1}
\end{array}\right)
$$$$
\text { or, in the form of double integrator, setting } Z_{X}=\left(\begin{array}{c}
q \\
\dot{q}
\end{array}\right)
$$

$$
Z_{X}=A_{X} Z_{X}+B_{X} \mathbf{u}_{X}
$$

where

$$
A_{X}=\left(\begin{array}{cc}
\bullet & \stackrel{\bullet}{\mathbf{I}} \\
-\widetilde{\mathbf{M}}^{-1} \mathbf{K} & -\widetilde{\mathbf{M}}^{-1} \mathbf{C}
\end{array}\right), B_{X}=\left(\begin{array}{cc}
\bullet & \bullet \\
0 & \widetilde{\mathbf{M}}^{-1} \mathbf{T}^{-1}
\end{array}\right)
$$

System(25) is equivalent to the following system, written according to cartesian coordinates using equations (1),

$$
A \ddot{\mathbf{X}}+B \dot{\mathbf{X}}+D \mathbf{X}+E=\mathbf{T}^{-1^{t}} \mathbf{u}_{X} \text {. }
$$

where

$A=\widetilde{\mathbf{M T}}, \mathbf{X}=\left(X_{1}, X_{2}, \ldots, X_{n}\right)^{t}, \quad B=\mathbf{C T}, \quad D=$ $\mathbf{K T}, \mathbf{X}_{\mathrm{e}}=\left(X_{1, e}, X_{2, e}, \ldots, X_{n, e}\right)^{t}$ and $E=\mathbf{K}\left(-\mathbf{T} \mathbf{X}_{e}+\mathbf{c}\right)$

Note that the forces due to virtual spring-damper elements guarantee the coordination of movement of the agents while they are in formation.

\section{4. $1 \mathrm{D}-1 \mathrm{D}^{\perp}$ FORMATION}

Let us consider now the problem of formation keeping in the plane. intuitively, the same questions pose : how to keep a specific configuration during movement and how to control the formation's size? As a response to these questions, we propose to break down the movement of the formation in the plane in two simultaneous movement following $X$ and $Y$ axes as shown in Fig.4. This approach allows to the formation to perform contractions and expansions in order to avoid potential obstacles ( see Fig.2).

Indeed, as the line formation of the first part can contract and expand horizontally, it was thought to develop a cross shaped formation to reproduce the behavior of the line formation according to the $Y$ axis. Therefore, we can ensure to increase

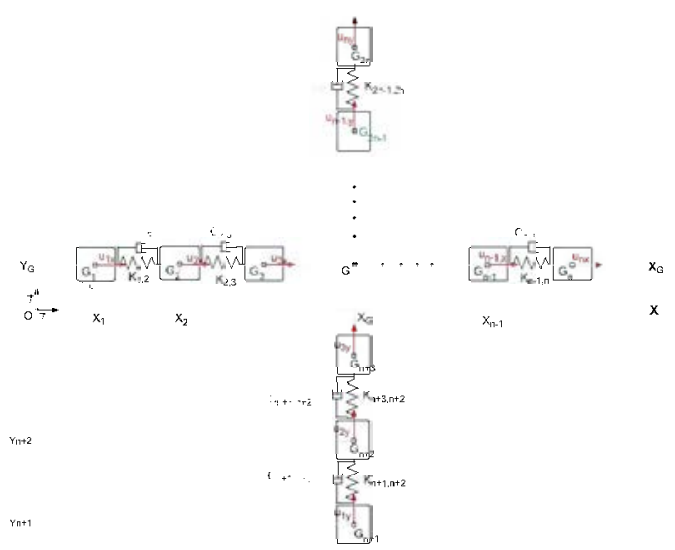

Fig. 4. $2 n$ cross-shaped formation

or decrease the size of the formation in a 2 dimension space. This new formation's configuration, consisting of two under formations admits a plane displacement. So, we get a crossshaped formation where the $n$ first vehicles are contracting and expanding only along the direction of abscissas while agents $n+1, \ldots, 2 n$ are doing the same in the $Y$ direction and by analogy to what was done in the first part they obey to the following dynamical system :

$$
\dot{Z}_{Y}=A_{Y} Z_{Y}+B_{Y} \mathbf{u}_{Y}
$$

With : $A_{Y}=\left(\begin{array}{cc}0 & \mathbf{I} \\ -\widetilde{\mathbf{M}}^{-1} \mathbf{K}_{Y} & -\widetilde{\mathbf{M}}^{-1} \mathbf{C}_{Y}\end{array}\right), B_{Y}=B_{X}$ $Z_{Y}=\left(\begin{array}{c}\mathbf{q}_{Y} \\ \mathbf{q}_{Y}\end{array}\right), \mathbf{q}_{Y}=\left(\begin{array}{c}q_{n+1} \\ q_{n+2} \\ \vdots \\ q_{2 n}\end{array}\right)=\left(\begin{array}{c}Y_{G} \\ y_{n+2}-y_{n+1} \\ \vdots \\ y_{2 n}-y_{2 n-1}\end{array}\right)$, $\mathbf{K}_{\mathbf{Y}}=\left(\begin{array}{cccc}0 & 0 & \cdots & 0 \\ 0 & K_{n+2, n+1} & \cdots & 0 \\ \bullet & \bullet & \ddots & 0 \\ \bullet & \bullet & \ddots & K_{2 n, 2 n-1}\end{array}\right)$ $\mathbf{C}_{Y}=\left(\begin{array}{cccc}0 & 0 & \cdots & 0 \\ 0 & C_{n+2, n+1} & \cdots & 0 \\ \bullet & \bullet & \ddots & 0 \\ \bullet & \bullet & \ddots & C_{2 n, 2 n-1}\end{array}\right)$ and $u_{Y^{-}}=\left(\begin{array}{c}u_{n+1} \\ u_{n+2} \\ \vdots \\ u_{2 n}\end{array}\right)$ Now, let $\mathbf{Z}=\left(\begin{array}{c}Z_{X} \\ Z_{Y}\end{array}\right)$,

This yields ultimately to the following differential system

$$
\dot{\mathbf{Z}}=\mathbf{A Z}+\mathrm{Bu} \text {. }
$$

with, $\mathbf{A}=\left(\begin{array}{cc}\mathbf{A}_{X} & 0 \\ 0 & \mathbf{A}_{Y}\end{array}\right)$ and $\mathrm{B}=\left(\begin{array}{cc}\mathrm{B}_{X} & 0 \\ 0 & \mathbf{B}_{Y}\end{array}\right)$

Recall that our goal is to model a formation of agents with a specific geometry. We know that whatever has to be the desired 
geometric configuration, it can always be defined by a set of nodes in the plan, or in other words by points of coordinates $(x, y)$ in the plan. Therefore one chooses to increase the size of our formation of agents by defining. as it has been done in the previous step which is actually a skeleton of departure for our final configuration. the coordinates of agents.

As a first step. we choose to add for example, another "line" to our formation. Fig. (5) shows the procedure of the formation extending. When the number of robots becomes very large, we can add another spring-damper elements along the $Y$ axis to prevent collision at infinity.

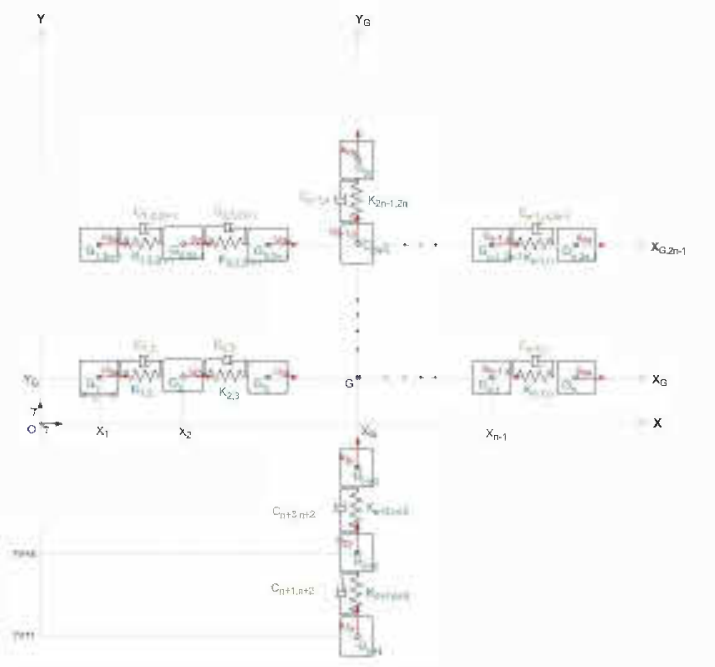

Fig. 5. Procedure to formation size increasing

\section{2D FORMATION}

Given any geometric configuration of a formation or desired shape of $n$ vehicles in the plane, we define a set of nodes $S$ (which regroup the cartesian coordinates of the vehicles in formation) :

$$
S=\left\{\left(x_{i}, y_{i}\right) ; i \in \mathcal{N}\right\}
$$

Where $\mathcal{N}=\{1, \ldots, n\}$.

We choose to separate the abscissas and the ordinates of these nodes which gives place to the following two sets :

$$
S_{1}=\left\{x_{i} ; i \in \mathcal{N}_{1}\right\}
$$

and

$$
S_{2}=\left\{y_{i} ; i \in \mathcal{N}_{2}\right\}
$$

$\mathcal{N}_{1}$ and $\mathcal{N}_{2}$ are, respectively, the sets of indexes relative to distinct abscissas and ordinates (see Fig.??). From $S_{1}$, we propose to generate a virtual structure, on the $X$ axis similar to the formation constrained to move in one direction. The same is done given $S_{2}$. The virtual structure, either along the $X$ or $Y$ axis is modeled as a series of masses linked by spring-damper elements. These structures, capable to stretch and compress. will guarantee that no collision can occur between the different robots during movement.

Thus, we can control the displacement of the vehicles by using the control forces acting on the abscissa and the ordinate of each robot. Consequently, we get a marionette like behavior. The formation of robots will behave as it was manipulated by virtual strings which are attached to each robot.

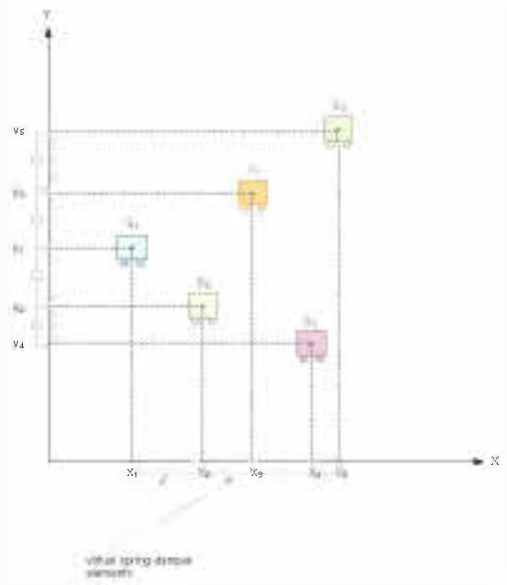

Fig. 6. virtual structures along $X$ and $Y$ axes

\subsection{Simulation results}

One verifies that a mobile robot cannot move away from the formation. Indeed, compared to an equilibrium configuration (a predefined desired shape), the move away of the entity belonging to the formation implies its return to the formation. since the latter is in equilibrium. Figures $8 \mathrm{a}, 8 \mathrm{~b}$ and $8 \mathrm{c}$ illustrate the process of recovering the equilibrium configuration after being object to an initial perturbation. If one affects to the formation a new equilibrium position. the disturbance of the formation will involve displacements of all the elements towards the new form of the equilibrium. Once the equilibrium is fixed, the displacement of the structure is asserted by its center of mass. As one took into account the dynamic, a formation through its flexible structure, can accelerate or decelerate while respecting the physical limits of each elements. However, each element of the formation can be the leader of all the structure according to the given instructions (as an example. robot 1 in Fig.9 is object to an external force forcing it to move along the $X$ axis and shove the other robots of the formation after recovering equilibrium configuration).

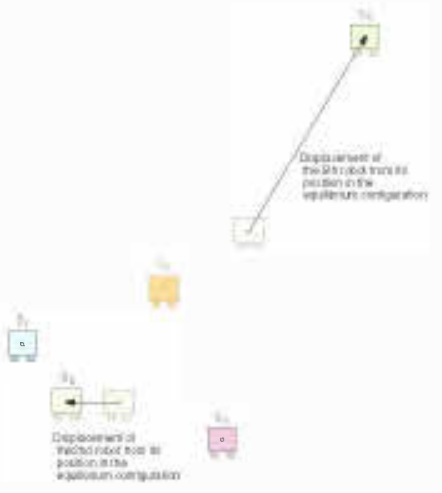

Fig. 7. Initial displacement of two robots of the formation from their equilibrium positions

\section{CONCLUSION}

In this work, we have proposed a flexible virtual structure in a 2D space for a formation of robots. By explicitly separating the 


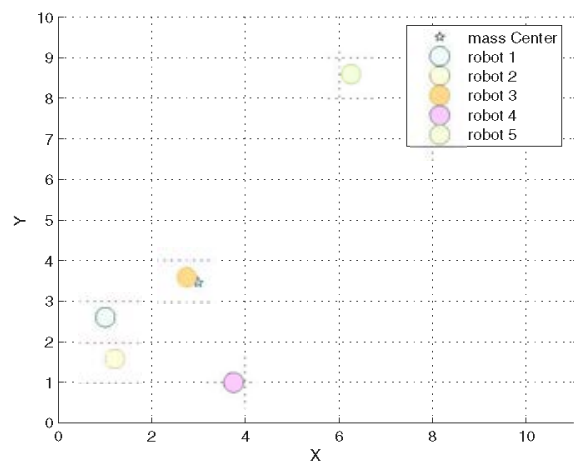

(a) screenshot 1 : Initial Perturbed configuration

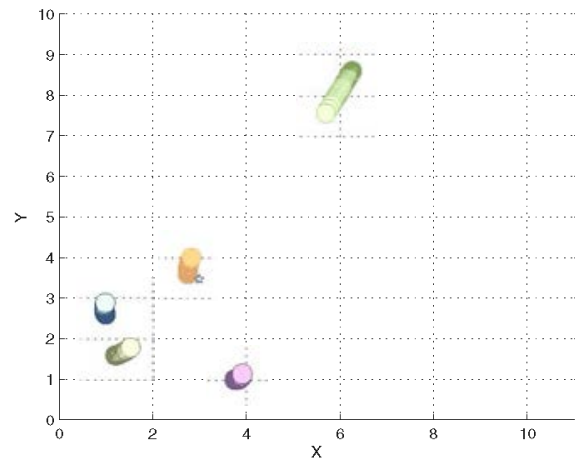

(b) screenshot 2 : Recovering desired configuration

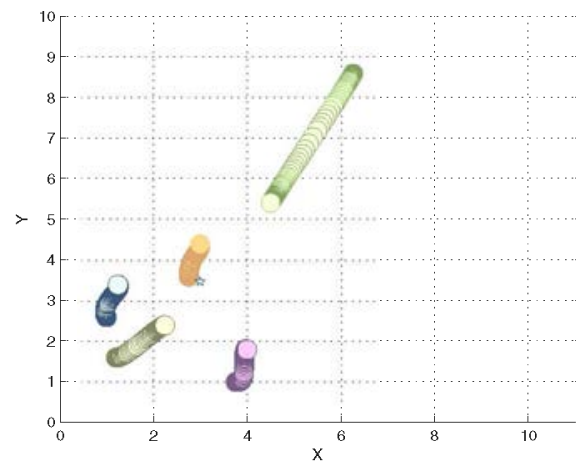

(c) screenshot 3 : Reaching desired equilibrium configuration

Fig. 8. Recovering desired configuration after a perturbation

flexibility into a contraction and expansion of the virtual structure on the cartesian axes, we developed equations of motion transforming the problem in the plane into a linear dynamic system. We have described a cross-shaped formation and how to increase its size by adding more robots. A general method suitable for any geometric desired configuration of the formation was presented. We have Implemented the method in a matlab program and showed that, the desired configuration, when perturbed gets back to the chosen equilibrium configuration and then continue its path (see Fig.9). The flexible character of the proposed formation, make easy the avoidance of an obstacle. This last idea is under development. In the same way, our investigations are towards a minimization of parameterizations of the system for a heterogeneous formation.

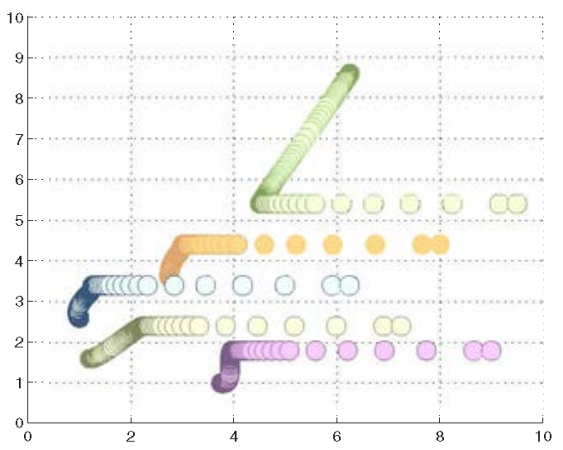

Fig. 9. Example of movement in the plane of a formation composed of five robots after recovering the desired configuration

\section{REFERENCES}

[1] M. Anthony Lewis And Kar-Han Tan. High Precision Formation Control of Mobile Robots Using Virtual Structures. Springer Netherlands, Autonomous Robots, Vol.4, pp.387-403, 1997.

[2] Tucker Balch and Ronald C. Arkin. Behavior-based Formation Control for Multi-robot Teams. IEEE Transactions on Robotics and Automation, Vol. 14, NO. 6, pp. 926-939, 1998.

[3] Wei Ren and Randal W. Beard. Virtual Structure Based Spacecraft Formation Control With Formation Feedback. AIAA Guidance, Navigation, and Control Conference and Exhibit,5-8 August 2002, Monterey, California.

[4] Sergio Monteiro and Estela Bicho. A Dynamical Systems Approach to Behavior-Based Formation Control. Proceedings of the 2002 IEEE International Conference on Robotics and Automation, Washington, DC May 2002.

[5] Roger Skjetne, Ivar-Andre Flakstad Ihle, and Thor I. Fossen. Formation control by synchronizing multiple maneuvering systems. Conference on Maneuvering and Control of Marine Crafts, IFAC MCMC'03, Girona, Spain, 2003.

[6] Wei Ren and Randal W. Beard. A decentralized scheme for spacecraft formation flying via the virtual structure approach. In A.F. Round, editor, AIAA Journal of Guidance, Control, and Dynamics, Vol.27, No.1, pp.73-82, 2004.

[7] Rafael E. Caicedo, John Valasek, and John L. Junkins Preliminary Results Of Vehicle Formation Control Using Dynamic Inversion. 42nd AIAA Aerospace Sciences Meeting and Exhibit.5 - 8 January 2004, Reno, Nevada.

[8] Emmett Lalish, Kristi A. Morgansen and Takashi Tsukamaki. Formation Tracking Control using Virtual Structures and Deconfliction. Proceedings of the IEEE Conference On Decision and Control, California, USA, 2006.

[9] Turker Sahin and Erkan Zergeroglu. Mobile Dynamically Reformable Formations for Efficient Flocking Behavior in Complex Environments. IEEE International Conference on Robotics and Automation,Pasadena, CA, USA, 2008. 